\title{
Direct observation of a Fermi surface and superconducting gap in $\mathrm{LuNi}_{2} \mathrm{~B}_{2} \mathrm{C}$
}

\author{
P. Starowicz, ${ }^{1,2}$ C. Liu, ${ }^{1}$ R. Khasanov, ${ }^{1,3}$ T. Kondo, ${ }^{1}$ G. Samolyuk, ${ }^{1}$ D. Gardenghi,${ }^{1,4}$ Y. Lee, ${ }^{1}$ T. Ohta, ${ }^{5}$ B. Harmon, ${ }^{1}$ \\ P. Canfield, ${ }^{1}$ S. Bud'ko, ${ }^{1}$ E. Rotenberg, ${ }^{5}$ and A. Kaminski ${ }^{1}$ \\ ${ }^{1}$ Ames Laboratory and Department of Physics and Astronomy, Iowa State University, Ames, Iowa 50011, USA \\ ${ }^{2}$ M. Smoluchowski Institute of Physics, Jagiellonian University, Reymonta 4, 30-059 Kraków, Poland \\ ${ }^{3}$ Physik-Institut der Universität Zürich, Winterthurerstrasse 190, CH-8057 Zürich, Switzerland \\ ${ }^{4}$ Bob Jones University, Greenville, South Carolina 29614, USA \\ ${ }^{5}$ Advanced Light Source, Berkeley National Laboratory, Berkeley, California 94720, USA
}

(Received 29 January 2008; published 30 April 2008)

\begin{abstract}
We measured the Fermi surface (FS), band dispersion, and superconducting gap in $\mathrm{LuNi}_{2} \mathrm{~B}_{2} \mathrm{C}$ using angle resolved photoemission spectroscopy. Experimental data were compared to the tight-binding version of the linear muffin-tin orbital (LMTO) method and linearized augmented plane-wave (LAPW) calculations. We found reasonable agreement between the two calculations and experimental data. The measured FS exhibits large parallel regions with a nesting vector that agrees with a previous positron annihilation study and calculations of the generalized susceptibility. The measured dispersion curves also agree reasonably well with the TB-LMTO calculations, although with some differences in the strength of the hybridization. In addition, the spectrum in the superconducting state revealed a $2 \mathrm{meV}$ superconducting gap. The data also clearly show the presence of a coherent peak above the chemical potential $\mu$, which originates from thermally excited electrons above the energy of $2 \Delta$. This feature was not previously observed in the Lu-based material.
\end{abstract}

DOI: 10.1103/PhysRevB.77.134520

PACS number(s): 74.70.Dd, 71.18.+y, 71.20.-b

\section{INTRODUCTION}

Rare earth nickel borocarbides $R \mathrm{Ni}_{2} \mathrm{~B}_{2} \mathrm{C}(R=$ rare earth) constitute an interesting class of materials, ${ }^{1-5}$ in which there is a competition and coexistence between superconductivity and magnetism. Among these compounds, nonmagnetic $\mathrm{LuNi}_{2} \mathrm{~B}_{2} \mathrm{C}$ has the highest superconducting critical temperature of $16.6 \mathrm{~K} .{ }^{1}$ The borocarbides exhibit a peculiar anisotropy of the superconducting gap, the character of which is still under debate. It is believed by various experimental and theoretical studies that the gap is highly anisotropic in the two nonmagnetic compounds $\mathrm{LuNi}_{2} \mathrm{~B}_{2} \mathrm{C}$ and $\mathrm{YNi}_{2} \mathrm{~B}_{2} \mathrm{C} .{ }^{6-14}$ Its symmetry was proposed to be $s+g,{ }^{9}$ which is consistent with certain experimental results, ${ }^{10}$ but an anisotropic $s$-wave symmetry has also been considered. ${ }^{6,11,13}$ Other experimental data indicate that the gap in $\mathrm{YNi}_{2} \mathrm{~B}_{2} \mathrm{C}$ has point nodes along the (100) and (010) directions. ${ }^{12} \mathrm{LuNi}_{2} \mathrm{~B}_{2} \mathrm{C}$ crystallizes in a body-centered tetragonal structure with lattice parameters $a$ $=3.4639 \AA$ and $c=10.6313 \AA .{ }^{15}$ Its crystal structure consists of Lu-C layers with $\mathrm{Ni}_{2} \mathrm{~B}_{2}$ sheets in between. Previous calculations revealed that $\mathrm{LuNi}_{2} \mathrm{~B}_{2} \mathrm{C}$ is characterized by a large density of states (DOS) at the Fermi energy $\left(E_{F}\right)$, which originate mainly, but not exclusively, from $\mathrm{Ni} d$ electrons. ${ }^{16-18}$ Another interesting feature is a flat band along the $\Gamma$ - $X$ direction just above $E_{F}$. The Fermi surface (FS) topography of $\mathrm{LuNi}_{2} \mathrm{~B}_{2} \mathrm{C}$ was studied by $a b$ initio calculations. ${ }^{19-21}$ Band structure calculations ${ }^{19}$ revealed a pronounced maximum in the generalized electronic susceptibility at $\left(\sim 0.6 a^{*}, 0,0\right)$, where $a^{*} \equiv 2 \pi / a$; this feature most likely arises from large nested regions of the FS. Moreover, phonon softening was observed in $\mathrm{LuNi}_{2} \mathrm{~B}_{2} \mathrm{C}$ by means of inelastic neutron scattering for a range of wave vectors around $\left(0.5 a^{*}, 0,0\right){ }^{22}$ Interestingly enough, the magnetic ordering, which was found in $R \mathrm{Ni}_{2} \mathrm{~B}_{2} \mathrm{C}$ compounds with magnetic atoms $R=\mathrm{Er}, \mathrm{Ho}, \mathrm{Tb}$, and $\mathrm{Gd}$, manifests a similar modulation vector, which is usually close to $\left(0.55 a^{*}, 0,0\right){ }^{4}$ The first experimental studies of the $\mathrm{LuNi}_{2} \mathrm{~B}_{2} \mathrm{C}\left(R \mathrm{Ni}_{2} \mathrm{~B}_{2} \mathrm{C}\right)$ Fermi surface were performed by means of two-dimensional angular correlation of electron-positron annihilation radiation (2D-ACAR) and the data were compared to the linear muffin-tin orbital (LMTO) and local density approximation (LDA) calculations. ${ }^{21}$ Nested parts of the FS were found with a nesting vector corresponding to both the phonon softening and the magnetic modulation vectors. The fraction of the FS participating in nesting was determined to be $4.4 \pm 0.5 \% .^{21}$ That study was, however, limited only to a rough "callipering" of the Fermi surface. We note that quantum oscillations were observed in de Haas-van Alphen measurements; ${ }^{23}$ however, the reconstruction of the Fermi surface shape is still lacking. Knowledge of the band structure, Fermi surface, and quasiparticle properties is deemed essential to understanding the interplay of the various interactions in these materials, as it may shed new light on other phenomena such as anisotropic superconductivity, the role of phonon softening, and the relationship between the superconductivity and magnetic ordering in borocarbides. It is also a prerequisite for a direct determination of the alleged anisotropy of the superconducting gap in these materials. In this report, we present angle resolved photoelectron spectroscopy (ARPES) measurements of the band dispersion, Fermi surface, and superconducting gap in the borocarbide with the highest $T_{c}, \mathrm{LuNi}_{2} \mathrm{~B}_{2} \mathrm{C}$. The experimental results were compared with the tight-binding LMTO (TB-LMTO) method and the full potential LAPW calculations. We found reasonable agreement with theory. The most significant difference between the calculations and experimental data is the strength of the hybridization. We also determined the averaged superconducting gap to be $\Delta_{0}=2.58 \mathrm{meV}$ (extrapolated for $T$ $=0 \mathrm{~K}$ ), which is in excellent agreement with the gap expected from the superconducting transition temperature 


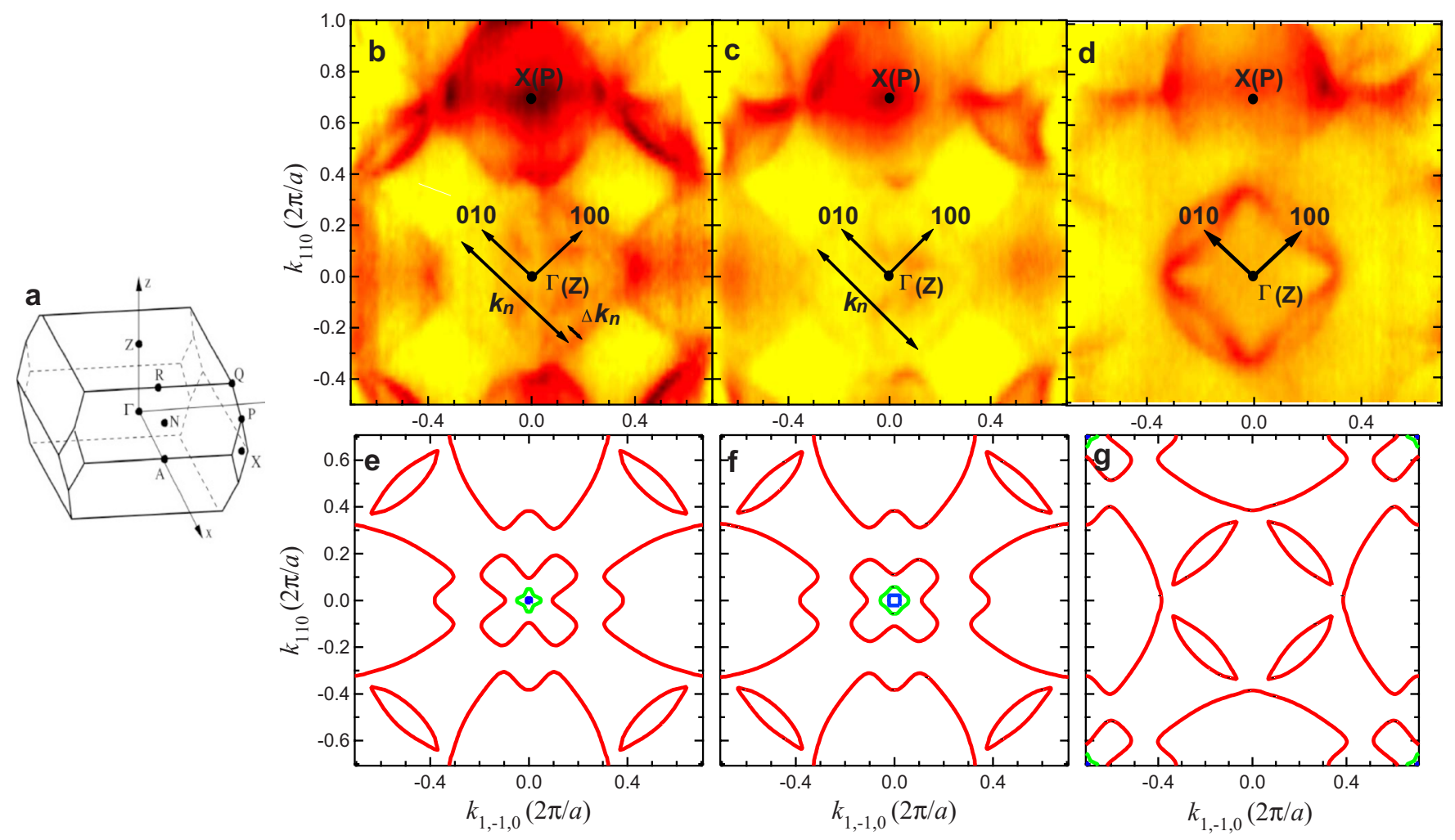

FIG. 1. (Color online) Comparison between the Fermi surface maps measured by ARPES and the linear muffin-tin orbital (TB-LMTO) calculation. (a) Sketch of the first Brillouin zone for $\mathrm{LuNi}_{2} \mathrm{~B}_{2} \mathrm{C}$. [(b)-(d)] ARPES mapping at the chemical potential for incident photon energies of $128.13 \mathrm{eV}, 119.44 \mathrm{eV}$, and $102.98 \mathrm{eV}$, respectively. [(e)-(g)] The Fermi surface maps obtained by TB-LMTO calculations for constant $k_{z}$ values equal to (e) 0.2 , (f) 0.15 , and (g) 0.8 expressed in the units of $\Gamma$ - $Z$ distance.

$\left(2 \Delta_{0} / k_{B} T_{c}=3.61\right)$ and an earlier electronic Raman scattering experiment. ${ }^{14}$

\section{EXPERIMENT}

$\mathrm{LuNi}_{2} \mathrm{~B}_{2} \mathrm{C}$ single crystals were grown at Ames Laboratory by means of a high-temperature flux technique. ${ }^{3,24}$ The platelike crystals were cleaved in situ at pressures better than 3 $\times 10^{-11}$ Torr $\left(4 \times 10^{-9} \mathrm{~Pa}\right)$ to reveal and maintain fresh $a-b$ surfaces. The Fermi surface and band structure mappings were performed at the 7.0.1 beamline at the Advanced Light Source using a Scienta R4000 analyzer. The energy and angle resolution were set at $\sim 30 \mathrm{meV}$ and $\sim 0.5^{\circ}$, respectively. The energy gap was measured with a Scienta 2002 analyzer and He-I photon source $(h \nu=21.2 \mathrm{eV})$, in which the overall energy resolution was set at $2 \mathrm{meV}$. The normal state data were measured at the Synchrotron Radiation Center using the PGM beamline and Scienta 2002 end station, with the energy and angular resolution set at $\sim 13 \mathrm{meV}$ and $0.25^{\circ}$, respectively. The data was measured over the whole momentum range presented in Figs. 1(b) -1 (d) on meshes of $856 \times 60$ points. It was then binned, interpolated, and truncated to the $350 \times 120$ size presented in Figs. 1(b)-1(d). Tight-binding linear muffin-tin orbital calculations were performed by the TB-LMTO program (version 47$)^{25}$ and the fullpotential linearized augmented plane-wave (LAPW) calculations were performed using the WIEN2K package. ${ }^{26}$

\section{RESULTS AND DISCUSSION}

Band structure and semiplanar Fermi surface cuts were determined for incident photon energies of 128.13, 119.44, and $102.98 \mathrm{eV}$ [Figs. 1(b)-1(d)], where the $\Gamma$ point in the Brillouin zone [Fig. 1(a)] corresponds to the normal emission of electrons along the (001) direction. A sin $k / k$ correction term ( $k$ being the $k$-space distance from the $\Gamma$ point) was also used to account for the mapping of the momentum space onto the angular distribution of photoelectrons. The ARPES process in three-dimensional (3D) materials leaves some ambiguity as to the $k_{z}$ component of the momentum (perpendicular to the sample surface), because it is not conserved in the photoemission process. This is due to a jump of the potential at the sample surface. From the conservation of energy and the remaining components of the momentum, one can calculate the relative changes in $k_{z}$ for various photon energies. To obtain the offset, one needs to seek guidance from the band structure calculations and identify the high symmetry points in the data. ${ }^{27}$ This allows estimation of the $k_{z}$ offset. The change in the wave vector component $k_{z}$ (parallel to the $c$ axis) between scans in Fig. 1(b) ( $h \nu$ $=128.13 \mathrm{eV})$ and Fig. 1(c) $(h \nu=119.44 \mathrm{eV})$ was calculated from momentum and energy conservation to be 0.33 of the $\Gamma-Z$ distance. Similarly, the change in the wave vector component $k_{z}$ between scans in Fig. 1(c) $(h \nu=119.44 \mathrm{eV})$ and Fig. 1(d) $(h \nu=102.98 \mathrm{eV})$ was 0.66 of the $\Gamma-Z$. The calculated Fermi surfaces were obtained for constant $k_{z}$ values by 

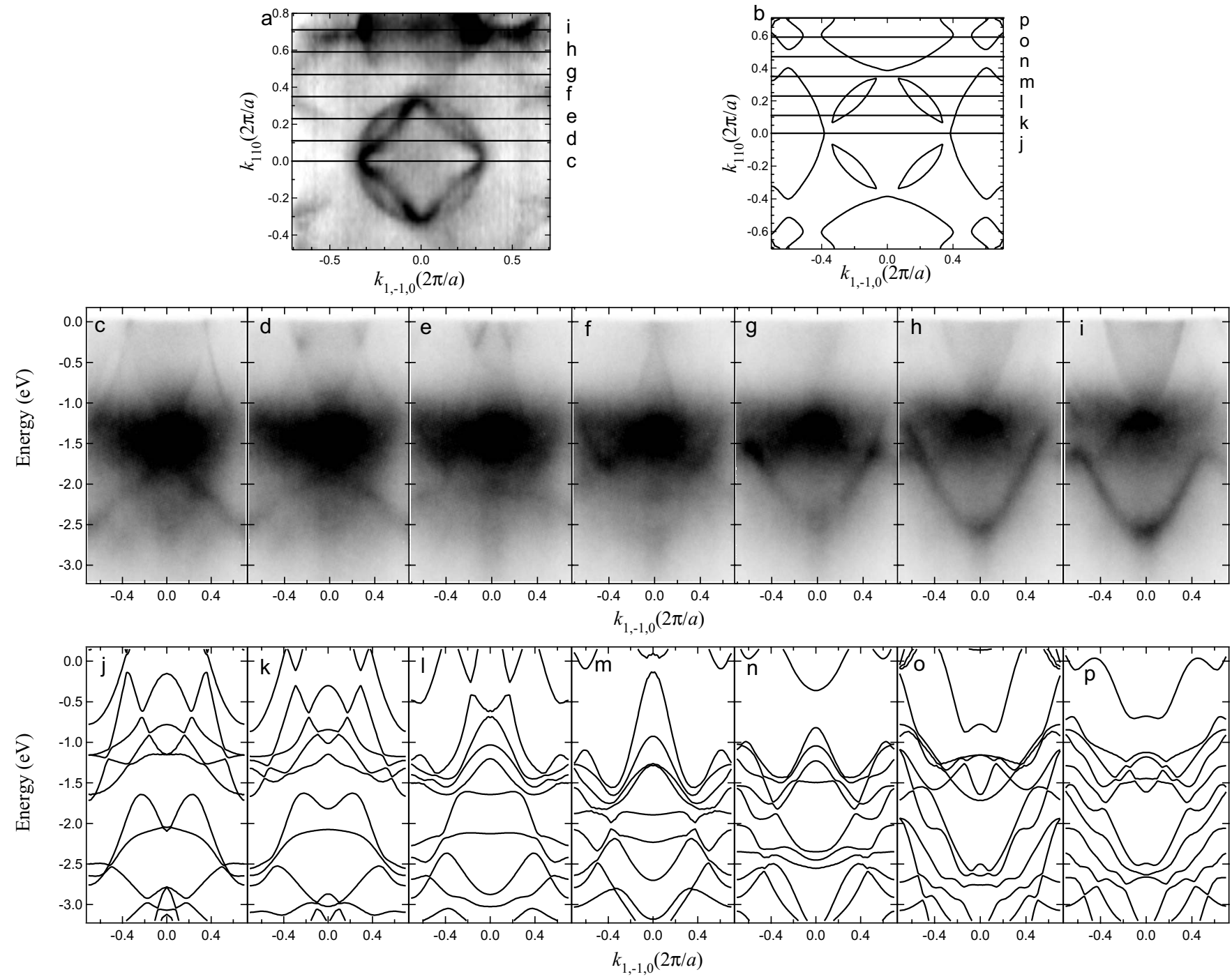

FIG. 2. Dispersion of the conduction bands obtained with a photon energy of $102.98 \mathrm{eV}$ [same as Fig. 1(d)], compared with the TB-LMTO calculation. (a) Fermi surface map with momentum cuts indicated by the solid lines, in which cuts (c) and (i) pass through the $\Gamma$ and $X$ points, respectively. (b) Fermi surface contours obtained by the TB-LMTO calculation for the value of $k_{z}$ corresponding to the data in panel (a). Panels (c)-(i): measured band dispersion along the cuts indicated in (a). Panels (j)-(p): calculated band dispersion along the cuts marked in panel (b).

means of the TB-LMTO method and are shown in Figs. $1(\mathrm{e})-1(\mathrm{~g})$. We estimated the values of the inner potential, $V_{0}=9.4 \mathrm{eV}$, and the work function, $\phi=4.6 \mathrm{eV}$, by comparing the high symmetry points between the calculated Fermi surfaces and the experimental data. This allowed us to determine the offset of the photon energy that corresponds to $k_{z}$ $=0$.

The Fermi surface maps for the incident photon energies of 128.13 and $119.44 \mathrm{eV}$ reveal large parallel parts of the FS with essentially the same nesting vector (spacing between the linear sections): $k_{n}=(0.59 \pm 0.04) a^{*}$ for Fig. 1(b) and $k_{n}$ $=(0.58 \pm 0.04) a^{*}$ for Fig. $1(\mathrm{c}) . k_{z}$ is expressed in the units of the $\Gamma-Z$ distance, where the $\Gamma$ point corresponds to $k_{z}=0$. Although the full 3D FS was not determined in great detail, a similar nesting vector was found for different $k_{z}$ values, which indicates that the FS likely has considerable nesting properties for a wide range of $k_{z}$ values. The constancy of the value of the nesting vector between $k_{z}=0.15$ and -0.2 is also consistent with results of calculations. The spacing between the parallel segments of the Fermi surface predicted by TBLMTO calculation is between $0.54 a^{*}$ and $0.55 a^{*}$. The LAPW calculation results (not shown) are $0.50 a^{*}$ and $0.57 a^{*}$, respectively. The detected $k_{n}$ is very close to the theoretically predicted value obtained from the generalized susceptibility. ${ }^{19}$ Our results also agree reasonably well with the nesting vector previously determined via 2D-ACAR. ${ }^{21}$

The Fermi surface map obtained at $102.98 \mathrm{eV}$ very closely resembles the calculated Fermi surface for $k_{z}=0.8 a^{*}$. The overall shapes of the measured and calculated Fermi surface sheets [Figs. 1(d) and $1(\mathrm{~g})$ ] are very similar. However, there is one significant difference. In the calculations, the four oval parts of the Fermi surface centered about $\Gamma-Z$ are well separated in momentum space [Fig. $1(\mathrm{~g})]$, while the data reveals that they are actually connected at the edges [Fig. 1(d)]. A lack of separation in the experimental data may indicate that the hybridization gap is overestimated in the 

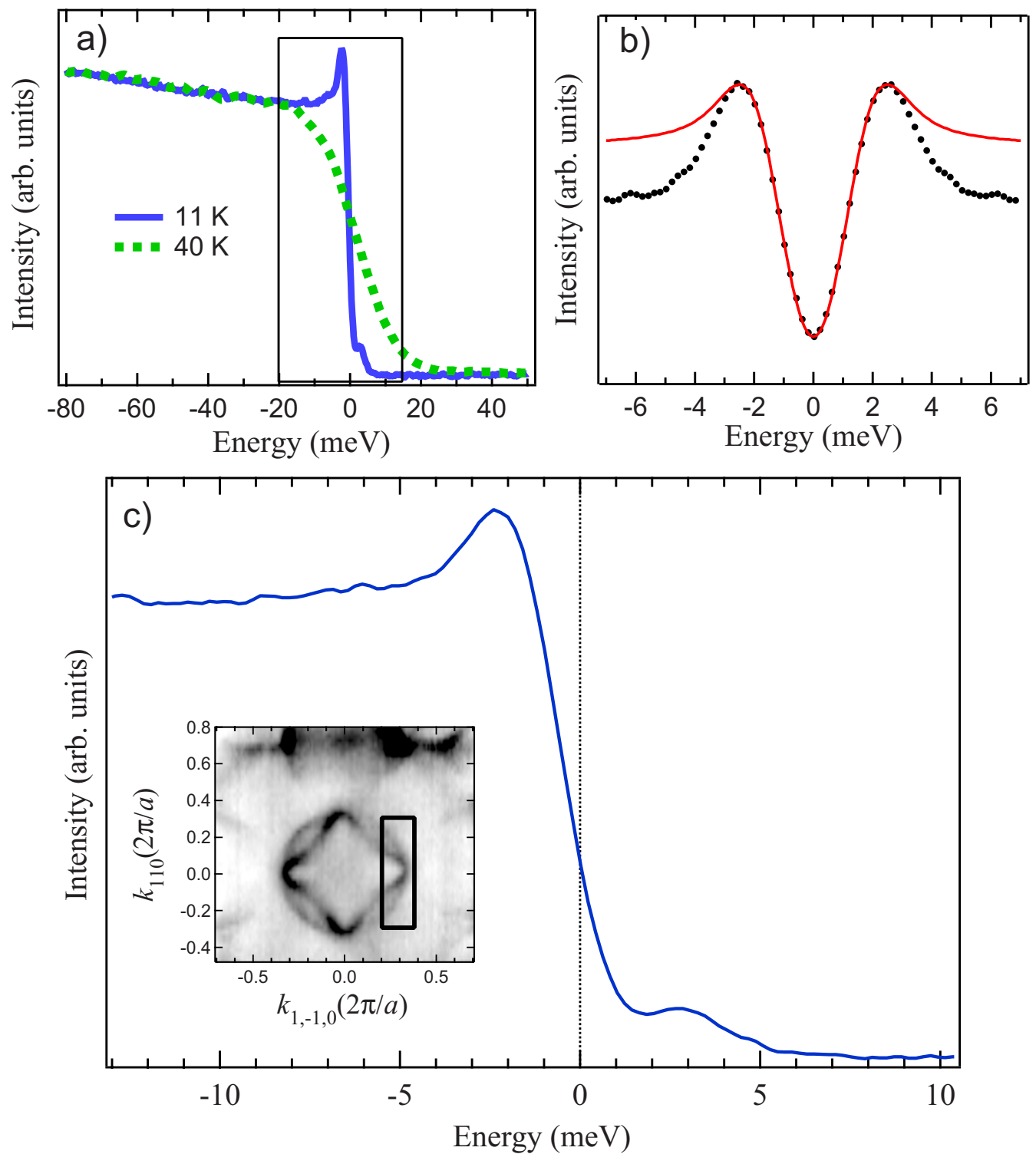

FIG. 3. (Color online) Superconducting gap of $\mathrm{LuNi}_{2} \mathrm{~B}_{2} \mathrm{C}$ measured in the $a-b$ plane (a) at $T=11 \pm 1 \mathrm{~K}$, compared with the normal state at $T=40 \mathrm{~K}$. (b) The Dynes function (solid red line) with the parameters $\Delta=1.5 \mathrm{meV}$ and $\Gamma=0.05 \mathrm{meV}$ fitted to the symmetrized spectrum (solid black circles). (c) Enlarged portion of superconducting spectra from (a) close to the chemical potential. The data was integrated over the momentum range shown in the inset.

calculations. These oval parts arise from the intersection of the electron and holelike bands. Interestingly enough, at the edges along the diagonal directions (e.g., 110) the bottom of the electron band and the top of the hole band appear to be pinned at the chemical potential, resulting in a characteristic "flower" shape.

In Fig. 2, we plot the band dispersion data along a few selected cuts in momentum space obtained at an incident photon energy of $102.98 \mathrm{eV}$ [Figs. 2(c)-2(i)], along with a calculated (TB-LMTO method) band dispersion for $k_{z}$ $\sim 0.8 a^{*}$ [Figs. 2(j)-2(p)]. The agreement between the measured and calculated band dispersion is rather good, especially in the proximity of the chemical potential. In the corresponding TB-LMTO calculations [Figs. 2(j)-2(p)], the same overall features are well reproduced, which shows the validity of the calculation in this material to a certain extent.
This agreement also validates the assignment of $k_{z}$ values to the cuts measured at various photon energies, which is very important when studying 3D materials with ARPES. The most significant difference is the hybridization gap, which is quite large in the calculations, but its signatures are, for the most part, absent in the measured data. For example, in Figs. 2(k) and 2(l) the high and low energy branches form hybridization gaps of about $200 \mathrm{meV}$ at $E=-0.3 \mathrm{eV}$, while in the corresponding measured data [Figs. 2(d) and 2(e)] the bands appear to disperse without a signature of the hybridization gap. One should consider if the observed features have any relation to the superconducting gap asymmetry and the observed phonon softening in $\mathrm{LuNi}_{2} \mathrm{~B}_{2} \mathrm{C}$. It should be noted that band structure calculations ${ }^{17,20}$ show that a flat band lies very close to, but slightly above, the Fermi level. This feature was unfortunately not observed in our data due to the 
Fermi function cutoff. However, a higher DOS near the Fermi level would explain the large number of scattered electrons observed with $k$ vectors along (110) and phonon softening for the discussed wave vectors. Consequently, this may lead to an anisotropy of the superconducting order parameter. This is in agreement with the results proposing that for $\mathrm{YNi}_{2} \mathrm{~B}_{2} \mathrm{C}$ the superconducting gap is larger just at (110) and diminishes or even has nodes along the (100) and (010) directions. ${ }^{12}$

Given the above concern, we measured the energy gap in $\mathrm{LuNi}_{2} \mathrm{~B}_{2} \mathrm{C}$ in the $a-b$ plane by partial angle-integrated photoelectron spectroscopy and compared it with the normal state Fermi surface. The opening of the superconducting gap is clearly shown in Fig. 3. In order to determine the magnitude of the gap, the Dynes function ${ }^{28}$ was fitted to the symmetrized $^{29}$ spectrum [Fig. 3(b)]. The fitted function yields the gap value of $\Delta=1.5 \mathrm{meV}$ for the sample at $T$ $=11 \mathrm{~K}$ with the $\Gamma$ parameter equal to $0.05 \mathrm{meV}$.

The striking feature in Fig. 3(c) is the pronounced peak above the chemical potential. This peak arises from thermal excitation of electrons above the $2 \Delta$. This points to high DOS just above $\mu$, which is consistent with the idea that the flat band along (110) direction, a large part of which is slightly over the Fermi energy, plays an important role in this anisotropic superconductivity. Similar peaks were recently reported in Y-based borocarbides. ${ }^{30}$ According to BCS theory, the energy gap value at zero temperature $\left(\Delta_{0}\right)$ is 2.52 $\mathrm{meV}$ for the $T_{c}=16.6 \mathrm{~K}$ superconductor. Our $\Delta$ value of 1.5 $\mathrm{meV}$ obtained at $T=11 \mathrm{~K}$ corresponds to $\Delta_{0}=2.58 \mathrm{meV}$, which is in good agreement with the BCS predictions, the electronic Raman scattering experiments, ${ }^{14}$ and the averaged value of the maximum and minimum gap obtained by pointcontact spectroscopy. ${ }^{8}$

\section{CONCLUSIONS}

We have performed measurements of the Fermi surface, band dispersion, and superconducting gap for highest $T_{c}$ rare earth nickel borocarbide superconductor $\mathrm{LuNi}_{2} \mathrm{~B}_{2} \mathrm{C}$. The experimental data were compared with two different density functional calculations. The overall agreement between theory and measurement is good. In the experiment, large parallel FS parts spaced with the vector $k_{n}=0.59 a^{*}$ have been found for two different incident photon energies, which is a confirmation of the previous theoretical predictions ${ }^{19}$ and earlier experimental studies. ${ }^{21}$ The calculated FS confirms the existence of large nested parts, with a nesting vector, which is in good agreement with the ARPES results presented here. The superconducting gap was measured and we also observed a coherent peak above the chemical potential. This peak arises due to electrons being thermally excited above the energy of $2 \Delta$.

\section{ACKNOWLEDGMENTS}

This work was supported by Director Office for Basic Energy Sciences, U.S. DOE. Work at Ames Laboratory was supported by the Department of Energy, Basic Energy Sciences under Contract No. DE-AC02-07CH11358. Advanced Light Source is operated by the U.S. DOE under Contract No. DE-AC03-76SF00098. Synchrotron Radiation Center is supported by the National Science Foundation under Award No. DMR-0537588. R.K. gratefully acknowledges support of the K. Alex Müller Foundation.
${ }^{1}$ R. J. Cava, H. Takagi, H. W. Zandbergen, J. J. Krajewski, W. F. Peck, Jr., T. Siegrist, B. Batlogg, R. B. van Dover, R. J. Felder, K. Mizuhashi, J. O. Lee, H. Eisaki, and S. Uchida, Nature (London) 367, 252 (1994).

${ }^{2}$ R. Nagarajan, C. Mazumdar, Z. Hossain, S. K. Dhar, K. V. Gopalakrishnan, L. C. Gupta, C. Godart, B. D. Padalia, and R. Vijayaraghavan, Phys. Rev. Lett. 72, 274 (1994).

${ }^{3}$ P. C. Canfield, P. L. Gammel, and D. J. Bishop, Phys. Today 51 (10), 40 (1998).

${ }^{4}$ K.-H. Müller and V. N. Narozhnyi, Rep. Prog. Phys. 64, 943 (2001).

${ }^{5}$ C. Mazumdar and R. Nagarajan, Curr. Sci. 88, 83 (2005).

${ }^{6}$ T. Yokoya, T. Kiss, T. Watanabe, S. Shin, M. Nohara, H. Takagi, and T. Oguchi, Phys. Rev. Lett. 85, 4952 (2000).

${ }^{7}$ E. Boaknin, R. W. Hill, C. Proust, C. Lupien, L. Taillefer, and P. C. Canfield, Phys. Rev. Lett. 87, 237001 (2001).

${ }^{8}$ N. L. Bobrov, S. I. Beloborod'ko, L. V. Tyutrina, I. K. Yanson, D. G. Naugle, and K. D. D. Rathnayaka, Phys. Rev. B 71, 014512 (2005).

${ }^{9}$ K. Maki, P. Thalmeier, and H. Won, Phys. Rev. B 65, 140502(R) (2002)

${ }^{10}$ P. Raychaudhuri, D. Jaiswal-Nagar, Goutam Sheet, S. Ramakrishnan, and H. Takeya, Phys. Rev. Lett. 93, 156802 (2004).
${ }^{11}$ P. Martinez-Samper, H. Suderow, S. Vieira, J. P. Brison, N. Luchier, P. Lejay, and P. C. Canfield, Phys. Rev. B 67, 014526 (2003).

${ }^{12}$ K. Izawa, K. Kamata, Y. Nakajima, Y. Matsuda, T. Watanabe, M. Nohara, H. Takagi, P. Thalmeier, and K. Maki, Phys. Rev. Lett. 89, 137006 (2002).

${ }^{13}$ A. Andreone, A. Cassinese, L. Gianni, M. Iavarone, F. Palomba, and R. Vaglio, Phys. Rev. B 64, 100505(R) (2001).

${ }^{14}$ In-Sang Yang, M. V. Klein, S. L. Cooper, P. C. Canfield, B. K. Cho, and Sung-Ik Lee, Phys. Rev. B 62, 1291 (2000).

${ }^{15}$ T. Siegrist, H. W. Zandbergen, R. J. Cava, J. J. Krajewski, and W. F. Peck, Jr., Nature (London) 367, 254 (1994).

${ }^{16}$ L. F. Mattheiss, Phys. Rev. B 49, 13279 (1994).

${ }^{17}$ W. E. Pickett and D. J. Singh, Phys. Rev. Lett. 72, 3702 (1994).

${ }^{18}$ R. Coehoorn, Physica C 228, 331 (1994).

${ }^{19}$ J. Y. Rhee, X. Wang, and B. N. Harmon, Phys. Rev. B 51, 15585 (1995).

${ }^{20}$ H. Kim, C.-D. Hwang, and J. Ihm, Phys. Rev. B 52, 4592 (1995).

${ }^{21}$ S. B. Dugdale, M. A. Alam, I. Wilkinson, R. J. Hughes, I. R. Fisher, P. C. Canfield, T. Jarlborg, and G. Santi, Phys. Rev. Lett. 83, 4824 (1999).

${ }^{22}$ P. Dervenagas, M. Bullock, J. Zarestky, P. Canfield, B. K. Cho, 
B. Harmon, A. I. Goldman, and C. Stassis, Phys. Rev. B 52, R9839 (1995).

${ }^{23}$ B. Bergk, O. Ignatchik, A. D. Bianchi, M. Jäckel, J. Wosnitza, J. Perenboom, and P. C. Canfield, Physica C 460-462, 630 (2007).

${ }^{24}$ P. C. Canfield and I. R. Fisher, J. Cryst. Growth 225, 155 (2001).

${ }^{25}$ O. Jepson and O. K. Andersen, Solid State Commun. 9, 1763 (1971); O. Jepsen and O. K. Andersen, Phys. Rev. B 29, 5965 (1984); P. E. Blöchl, O. Jepsen, and O. K. Andersen, ibid. 49, 16223 (1994).

${ }^{26}$ P. Blaha, K. Schwarz, G. Madsen, D. Kvasnicka, and J. Luitz, WIEN2k, An Augmented Plane Wave Plus Local Orbitals Program for Calculating Crystal Properties (Karlheinz Schwarz, Technical University Wien, Austria, 2001).
${ }^{27}$ S. Hufner, Photoelectron Spectroscopy (Springer, Berlin, 1995), pp. 268-270.

${ }^{28}$ R. C. Dynes, V. Narayanamurti, and J. P. Garno, Phys. Rev. Lett. 41, 1509 (1978).

${ }^{29}$ M. R. Norman, H. Ding, M. Randeria, J. C. Campuzano, T. Yokoya, T. Takeuchi, T. Takahashi, T. Mochiku, K. Kadowaki, P. Guptasarma, and D. G. Hinks, Nature (London) 392, 157 (1998).

${ }^{30}$ T. Baba, T. Yokoya, S. Tsuda, T. Kiss, T. Shimojima, K. Ishizaka, H. Takeya, K. Hirata, T. Watanabe, M. Nohara, H. Takagi, N. Nakai, K. Machida, T. Togashi, S. Watanabe, X.-Y. Wang, C. T. Chen, and S. Shin, Phys. Rev. Lett. 100, 017003 (2008). 